\title{
Avaliação do consumo de álcool entre adolescentes e os problemas associados*
}

\author{
Nayara Pires Nadaleti ${ }^{1}$ \\ Eliene Sousa Muro ${ }^{1}$ \\ Camila Csizmar de Carvalho ${ }^{1}$ \\ Bianca Bacelar de Assis ${ }^{1}$ \\ Denis Moreira da Silva ${ }^{1}$ \\ Erika de Cássia Lopes Chaves ${ }^{1}$
}

Objetivo: investigar o consumo de álcool por adolescentes e os problemas associados ao mesmo. Métodos: estudo descritivo e transversal, realizado com 209 alunos do ensino fundamental II. Coleta de dados: aplicou-se um Formulário de avaliação do perfil sociodemográfico, dados antropométricos, Drug Use Screening Inventory. Resultados: o consumo de álcool foi na mesma proporção entre meninos e meninas com idade superior a 13 anos. Os adolescentes que utilizaram álcool apresentaram maior probabilidade de se envolverem em situações de risco, como acidentes automobilísticos e dificuldades para resistir ao uso de outras drogas. Conclusão: o álcool causa efeitos negativos à vida do adolescente e a influência de amigos e de familiares pode potencializar seu consumo.

Descritores: Adolescentes; Consumo de Álcool por Menores; Estado Nutricional; Estudantes; Enfermagem.

\footnotetext{
* Apoio Financeiro da Fundação de Amparo à Pesquisa do Estado de Minas Gerais (FAPEMIG), Brasil, processo $\mathrm{n}^{\circ} \mathrm{APQ}$ 02909-12.

${ }^{1}$ Universidade Federal de Alfenas, Alfenas, MG, Brasil.
} 


\section{Assessment of alcohol consumption among adolescents}

\section{and the associated problems}

Objective: investigating the consumption of alcohol by youths and also its problems and evaluating the relation between both of them. Method: descriptive study and transversal, sample 209 students from elementary school. It was applied an evaluation form about the sociodemographic profile, anthropometric, Drug Use Screening Inventory. Results: the consumption was in the same proportion among boys and girls and older than 13 years old. Adolescents who used alcohol were more likely to be involved in situations of risk, such as car accidents and difficulties to resist the use of other drugs. Conclusion: alcohol causes negative effects to adolescent life and the influence of friends and family can boost their consumption.

Descriptors: Adolescents; Alcohol Consumption by Minors; Nutritional Status; Students; Nursing.

\section{Evaluación del consumo de alcohol entre los adolescentes y los problemas asociados}

Objetivo: investigar el consumo de alcohol por adolescentes y problemas decurrentes del mismo y avaliar la relacion entre ambos. Métodos: estudio descriptivo y transversal, muestra de 209 alumnos de enseñanza fundamental II. Colecta de datos se aplicou un Formulário de avaliacion del perfil sociodemográfico, antropométricos, Drug Use Screening Inventory. Resultados: el consumo de alcohol estaba en la misma proporción entre los niños y niñas de más de 13 años de edad. Los adolescentes que usan el alcohol eran más propensos a participar en situaciones de riesgo, tales como los accidentes de tráfico y difícil de resistir el uso de otras drogas. Conclusión: el alcohol provoca efectos negativos en la vida de los adolescentes y la influencia de los amigos y la familia puede aumentar su consumo.

Descriptores: Adolescentes; Consumo de Alcohol en Menores; Estado Nutricional; Estudiantes; Enfermeria.

Introdução

O consumo de álcool e de outras drogas é um problema crescente de saúde pública no Brasil e no mundo e ocorre cada vez mais em idade precoce, sendo o álcool a droga apontada em estudos como a mais consumida pelos adolescentes ${ }^{(1-2)}$.
Os fenômenos relacionados ao uso de álcool na adolescência são complexos e envolvem aspectos psíquicos, emocionais, cognitivos e sociais ${ }^{(3)}$. Essa fase é marcada pelo desenvolvimento individual em que as transformações biopsicossociais são vivenciadas pelos jovens muitas vezes de forma conflituosa, tornando-se estes vulneráveis e expostos a riscos. 
Frente às mudanças decorrentes dessa faixa etária, o adolescente inicia o uso de bebidas alcóolicas para uma aceitação coletiva, na busca da identidade pessoal e da independência(3).

O primeiro contato com o álcool ocorre, geralmente, na própria residência, de modo a imitar a conduta de familiares ${ }^{(4)}$ ou pelo fato de possuírem amigos que consomem a droga. Os amigos exercem grande influência sobre o comportamento do jovem, influenciando-os a utilizarem essa substância para se inserirem no grupo e criarem certa popularidade ${ }^{(1)}$.

No que diz respeito ao consumo por parte de familiares, pais que bebem possuem dificuldade de dialogar sobre o tema quando se deparam com a necessidade de prevenir o uso precoce da substância pelos filhos, uma vez que o uso do álcool pelos pais induz e facilita tal conduta pelos adolescentes ${ }^{(5)}$.

O uso dessa substância na adolescência influencia negativamente o comportamento dos jovens ${ }^{(2)}$ e está relacionado diretamente com múltiplos prejuízos que interferem no desenvolvimento do indivíduo, aumentando a probabilidade de envolvimento em brigas, de acidentes automobilísticos, de conflitos familiares, de evasão escolar, de déficits no desempenho escolar, de graves complicações para a saúde, de atividade sexual em situação de risco, dentre outros ${ }^{(6-7)}$.

Além das consequências supracitadas, acarretadas pelo uso do álcool, estas possuem diferentes efeitos no corpo humano; dentre estes, sua contribuição tanto para a obesidade, para o ganho de medidas antropométricas, de peso e de gordura quanto para a desnutrição proteicocalórica, dependendo da intensidade do consumo ${ }^{(8)}$.

Dessa forma, justifica-se este estudo pela necessidade de aquisição de dados atualizados a respeito do consumo de álcool por adolescentes, bem como, reforçar o conhecimento acerca dos danos causados por este consumo nessa faixa etária, de modo que contribua para a formulação de estratégias de prevenção do consumo de álcool que permita instrumentar os profissionais de saúde, sobretudo o enfermeiro atuante na saúde coletiva, para melhor assistir os jovens com essa problemática.

Assim, este estudo teve como objetivo investigar o consumo de álcool por adolescentes e os problemas associados ao mesmo.

\section{Metodologia}

Trata-se de um estudo descritivo, transversal, de abordagem quantitativa, realizado com estudantes dos sétimos e oitavos anos do Ensino Fundamental II em uma escola pública situada no Sul do Estado de Minas Gerais.

Todos os alunos (N: 250) foram convidados a participar do estudo e a amostra foi composta por 209 $(83,6 \%)$ voluntários. Os critérios de elegibilidade foram: estarem regularmente matriculados nos sétimos ou oitavos anos e estarem presentes em sala de aula no momento da coleta de dados, sendo excluídos aqueles que, após três tentativas de contatos, não foram encontrados.

A coleta de dados foi realizada entre os meses de maio e agosto de 2013 e foram utilizados dois instrumentos: Formulário de avaliação do perfil sociodemográfico e dos dados antropométricos dos participantes e o DUSI (Drug Use Screening Inventory) para investigação do perfil de consumo de álcool.

O DUSI foi desenvolvido originalmente nos Estados Unidos da América (EUA) ${ }^{(9)}$ e posteriormente adaptado e validado para a língua portuguesa ${ }^{(10)}$. Trata-se de um questionário composto por uma tabela inicial que aborda a frequência de consumo no último mês de treze classes de substâncias psicoativas, seguida por 159 questões divididas em 10 áreas, que fornecem um perfil da intensidade de problemas em relação ao uso de substância; comportamento; saúde; transtornos psiquiátricos; sociabilidade; sistema familiar; escola; trabalho; relacionamento com amigos e lazer e recreação.

O uso do DUSI para a triagem de tipos específicos de drogas tem sido observado em diferentes pesquisas ${ }^{(1,11)}$, a fim de avaliar, por exemplo, apenas o uso dessas substâncias no último mês, apenas seu nível de consumo ou, ainda, investigar a relação do uso de drogas com o desempenho escolar.

Assim, no presente estudo, foi realizado um recorte do instrumento, em que utilizou-se as respostas referentes à frequência apenas do consumo de álcool. Para investigar os problemas associados ao consumo dessa substância foram utilizados os domínios: uso de substâncias, comportamento, saúde, transtornos psiquiátricos, sistema familiar, escola, relacionamento com amigos e lazer e recreação. 
Para os procedimentos de coleta de dados, os instrumentos de pesquisa foram colocados em envelopes e entregues aos alunos no começo das atividades didáticas e recolhidos ao final das mesmas, já preenchidos.

A avaliação nutricional antropométrica dos adolescentes foi previamente agendada na escola em estudo. Para a aferição do peso, foi utilizada balança digital portátil Techine ${ }^{\circledR}$ Mod. BAL-180 BR, com graduação de $100 \mathrm{~g}$ e capacidade de $180 \mathrm{Kg}$ e solicitado ao indivíduo que mantivesse posição ortostática, ereto, braços estendidos ao longo do corpo, pés juntos, posicionado no centro da balança.

Para a aferição da estatura, foi utilizado um estadiômetro de madeira Altura Exata $®$, altura máxima $213 \mathrm{~cm}$, cujos escores de classificação, por idade, e os pontos de corte foram os preconizados pela Organização Mundial da Saúde (OMS), em 2007; da mesma forma, o diagnóstico do crescimento, que foi dado por meio de percentil e escore $Z^{(12)}$.

O Índice de Massa Corporal (IMC) foi calculado pelo peso dado em quilogramas $(\mathrm{kg})$ e a estatura em metros $(m)$, elevado ao quadrado, resultando em um valor expresso em $\mathrm{Kg} / \mathrm{m}^{2}$, observando os critérios de avaliação do estado nutricional do adolescente, estabelecidos pela $\mathrm{OMS}^{(12)}$.

Foi realizado o cálculo amostral em que considerou a margem de erro de $2,7 \%$ e $95 \%$ de confiança. Para análise dos dados utilizou-se o programa estatístico Statistical Package for the Social Sciences (SPSS), versão 20,0 em que foi empregada a estatística descritiva a fim de resumir e apresentar os dados obtidos. As variáveis nominais foram descritas pela análise de frequência e o cruzamento de variáveis foi realizado usando uma tabela de contingência, também calculou-se o teste qui-quadrado ou teste Exato de Fisher usando $5 \%$ de significância, e o odds ratios (OR) levando em consideração intervalo do confiança (IC) de 95\%.
Para assegurar os direitos dos participantes e cumprir os aspectos éticos, este estudo foi aprovado pelo Comitê de Ética em Pesquisa, por meio da Plataforma Brasil, sob parecer número 238.188, no ano de 2013.

Vale ressaltar que, os pais/responsáveis e os adolescentes que concordaram com a participação no referido estudo assinaram o Termo de Consentimento Livre e Esclarecido e o Termo de Assentimento, respectivamente.

\section{Resultados}

Dos 209 alunos que participaram deste estudo, $50,7 \%$ eram do sexo feminino e $49,3 \%$, do masculino. A média de idade encontrada foi de 13 anos, com variação entre 11 e 17 anos. Considerando os sétimos anos, a média de idade foi 12,7 anos $(n=102)$, enquanto nos oitavos, 13,5 anos ( $n=107)$.

A baixa condição socioeconômica foi observada neste estudo, uma vez que $32 \%(n=66)$ dos adolescentes relataram renda familiar menor que um salário mínimo, $62 \%(n=130)$ de um a cinco salários mínimos e apenas $6 \%(n=13)$ possuíam uma renda familiar acima de cinco salários. Adicionalmente, a maioria, 73,2\% ( $n=153)$ possui formação familiar de mais de quatro indivíduos.

A investigação do perfil do uso de álcool demonstrou que, dos 209 adolescentes, $21 \%$ ( $n=44)$ utilizaram esta substância nos últimos 30 dias que antecederam a entrevista. A Tabela 1 apresenta informações sobre o uso de álcool por faixa etária e por sexo, em que se observa a ausência de significância estatística na comparação entre o uso de álcool e o sexo, além de este não ter representado um fator de risco, ou seja, indivíduos do sexo feminino e masculino utilizaram álcool na mesma proporção. Por outro lado, a idade foi estatisticamente significativa, uma vez que os adolescentes acima de 13 anos consomem mais álcool.

Tabela 1 - Frequência do consumo de álcool por faixa etária e sexo (n=209). Alfenas, MG, Brasil, 2013

\begin{tabular}{|c|c|c|c|c|c|c|c|}
\hline & \multirow{3}{*}{ Variável } & \multicolumn{4}{|c|}{ Uso de álcool } & \multirow{3}{*}{$\begin{array}{c}\text { OR bruto IC } \\
(95 \%)\end{array}$} & \multirow{3}{*}{ Valor $\mathbf{P}$} \\
\hline & & \multicolumn{2}{|c|}{ Não } & \multicolumn{2}{|c|}{ Sim } & & \\
\hline & & $\mathbf{n}$ & $\%$ & $\mathbf{n}$ & $\%$ & & \\
\hline \multirow[b]{2}{*}{ Sexo } & Feminino & 84 & $79,2 \%$ & 22 & $20,8 \%$ & 1,037 & \multirow[b]{2}{*}{$0,915^{*}$} \\
\hline & Masculino & 81 & $78,6 \%$ & 22 & $21,4 \%$ & $(0,533 ; 2,017)$ & \\
\hline \multirow{2}{*}{ Idade } & $\leq 13$ anos & 128 & $85,9 \%$ & 21 & $14,1 \%$ & 3,789 & \multirow{2}{*}{$<0,001^{*}$} \\
\hline & $>13$ anos & 37 & $61,7 \%$ & 23 & $38,3 \%$ & $(1,890 ; 7,596)$ & \\
\hline
\end{tabular}

Fonte: Dados da pesquisa. *Teste Qui-Quadrado 
A maioria dos adolescentes $(98,1 \%)$ conhecem os problemas causados pelo uso do álcool e já foram orientados ( $77 \%$ ) quanto a isso. Contudo, ao identificar o risco de esses jovens se envolverem em diferentes eventos nocivos, decorrentes do uso do álcool, foi verificado que quem consome teve maior risco, conforme apresentam as tabelas seguintes.

De acordo com exposto na Tabela 2, os jovens que consumiam álcool tiveram riscos elevados de se envolverem em acidentes automobilísticos e dificuldades para resistir ao uso de outras drogas. Por outro lado, os jovens que não utilizaram álcool, apresentaram menor probabilidade de exibirem maus comportamentos, de se envolver em brigas e em atividades arriscadas, como ter relações sexuais em situação de risco, além de apresentar menos problemas de saúde como transtornos psiquiátricos.

Tabela 2 - Implicações decorrentes do uso ou não uso de álcool pelos adolescentes $(n=209)$. Alfenas, MG, Brasil, 2013

\begin{tabular}{|c|c|c|c|c|c|c|c|}
\hline \multirow{3}{*}{ Fatores } & & \multicolumn{4}{|c|}{ Uso de álcool } & \multirow{3}{*}{$\begin{array}{l}\text { OR bruto IC } \\
\qquad(95 \%)\end{array}$} & \multirow{3}{*}{ Valor $\mathbf{P}$} \\
\hline & & \multicolumn{2}{|c|}{ Não } & \multicolumn{2}{|c|}{ Sim } & & \\
\hline & & $\mathbf{n}$ & $\%$ & $\mathbf{n}$ & $\%$ & & \\
\hline \multicolumn{8}{|c|}{ Problemas relacionados ao uso de substâncias } \\
\hline \multirow{2}{*}{$\begin{array}{l}\text { Efeito do álcool sobre a } \\
\text { memória }\end{array}$} & Sim & 2 & 20 & 8 & 80 & 0,055 & \multirow{2}{*}{$<0,001^{*}$} \\
\hline & Não & 163 & 81,9 & 36 & 18,1 & $(0,011 ; 0,271)$ & \\
\hline \multirow{2}{*}{$\begin{array}{l}\text { Envolvimento em } \\
\text { acidente de carro }\end{array}$} & Sim & 0 & 0 & 3 & 100 & 5,024 & \multirow{2}{*}{$0,009^{*}$} \\
\hline & Não & 165 & 80,1 & 41 & 19,9 & $(3,820 ; 6,608)$ & \\
\hline \multicolumn{8}{|c|}{ Alterações de comportamento } \\
\hline \multirow{2}{*}{$\begin{array}{l}\text { Envolvimento com } \\
\text { brigas }\end{array}$} & Sim & 30 & 61,2 & 19 & 38,8 & 0,292 & \multirow{2}{*}{$0,001^{\dagger}$} \\
\hline & Não & 135 & 84,4 & 25 & 15,6 & $(0,143 ; 0,598)$ & \\
\hline \multirow{2}{*}{ Agressividade } & Sim & 6 & 42,9 & 8 & 57,1 & 0,170 & \multirow{2}{*}{$0,002^{*}$} \\
\hline & Não & 159 & 81,5 & 36 & 18,5 & $(0,055 ; 0,520)$ & \\
\hline \multirow{2}{*}{ Atitudes arriscadas } & Sim & 21 & 52,5 & 19 & 47,5 & 0,192 & \multirow{2}{*}{$<0,001^{\dagger}$} \\
\hline & Não & 144 & 85,2 & 44 & 21,1 & $(0,090 ; 0,407)$ & \\
\hline \multicolumn{8}{|c|}{ Problemas relacionados à saúde } \\
\hline \multirow{2}{*}{$\begin{array}{l}\text { Relações sexuais em } \\
\text { situação de risco }\end{array}$} & Sim & 2 & 28,6 & 5 & 71,4 & 0,096 & \multirow{2}{*}{$0,005^{*}$} \\
\hline & Não & 163 & 80,7 & 39 & 19,3 & $(0,018 ; 0,512)$ & \\
\hline \multicolumn{8}{|c|}{ Problemas relacionados a transtornos psiquiátricos } \\
\hline \multirow{2}{*}{$\begin{array}{l}\text { Problemas de } \\
\text { concentração }\end{array}$} & Sim & 46 & 69,7 & 20 & 30,3 & 0,464 & \multirow{2}{*}{$0,026^{\dagger}$} \\
\hline & Não & 119 & 83,2 & 24 & 16,8 & $(0,234 ; 0,919)$ & \\
\hline \multirow{2}{*}{ Sentimento de tristeza } & Sim & 55 & 70,5 & 23 & 29,5 & 0,457 & \multirow{2}{*}{$0,021^{\dagger}$} \\
\hline & Não & 110 & 84 & 21 & 16 & $(0,233 ; 0,896)$ & \\
\hline \multirow{2}{*}{ Instabilidade do humor } & Sim & 105 & 73,9 & 37 & 26,1 & 0,331 & \multirow{2}{*}{$0,010^{\dagger}$} \\
\hline & Não & 60 & 89.6 & 7 & 10,4 & $(0,139 ; 0,789)$ & \\
\hline
\end{tabular}

Fonte: Dados da Pesquisa. *Teste Exato de Fisher; †Teste de Qui-quadrado

A Tabela 3 aponta as implicações decorrentes do uso ou não uso de álcool pelos adolescentes no sistema familiar, no rendimento escolar, na relação com os amigos, e nas atividades de lazer e de recreação. Pais ou responsáveis presentes em casa a maior parte do tempo, que conhecem o cotidiano e lugares em que os filhos frequentam, e que não se envolvem em discussões familiares, conferiram um fator de proteção, reduzindo os riscos para se envolverem com bebidas alcoólicas. Ainda, aqueles adolescentes que não consumiam álcool tiveram menos chances de ter problemas relacionados à escola, aos amigos e ao lazer, como frequentar lugares sem conhecimento dos pais, possuir amigos que consomem ou comercializam álcool e outras drogas, e que se envolveram em roubos ou danos a patrimônios públicos ou privados.
No que diz respeito ao consumo de álcool pelos familiares, $48,8 \%$ ( $n=102)$ dos alunos afirmaram que os moradores da casa fazem uso de bebidas alcoólicas; dentre estes, destacam-se o pai, a mãe, os irmãos, os tios e os avós.

Com relação ao estado nutricional antropométrico dos adolescentes, a média do peso encontrada foi de $49,7 \mathrm{Kg}$ e da altura, 1,58 metros, sendo a média de IMC $19,7 \mathrm{Kg} / \mathrm{m} 2$, com mínima de $12,4 \mathrm{Kg} / \mathrm{m} 2$ e máxima de $35,5 \mathrm{Kg} / \mathrm{m} 2$. A Tabela 4 apresenta a classificação do estado nutricional antropométrico, variáveis relacionadas a peso, altura e IMC, relacionado com o uso e não uso de álcool pelos adolescentes.

Pode-se observar que não houve significância estatística $(p>0,332)$ para a correlação entre uso e não uso do álcool com o estado nutricional antropométrico dos adolescentes investigados. 
Tabela 3 - Implicações decorrentes do uso ou não uso de álcool pelos adolescentes ( $n=209)$. Alfenas, MG, Brasil, 2013

\begin{tabular}{|c|c|c|c|c|c|c|c|}
\hline \multirow{3}{*}{ Fatores } & & \multicolumn{4}{|c|}{ Uso de álcool } & \multirow{3}{*}{$\begin{array}{l}\text { OR bruto IC } \\
(95 \%)\end{array}$} & \multirow{3}{*}{ Valor $\mathbf{P}$} \\
\hline & & \multicolumn{2}{|c|}{ Não } & \multicolumn{2}{|c|}{ Sim } & & \\
\hline & & $\mathbf{n}$ & $\%$ & $\mathbf{n}$ & $\%$ & & \\
\hline \multicolumn{8}{|c|}{ Problemas relacionados ao sistema familiar } \\
\hline \multirow{2}{*}{ Discussões com familiares } & Sim & 22 & 61,1 & 14 & 38,9 & 0,330 & \multirow{2}{*}{$0,004^{*}$} \\
\hline & Não & 143 & 82,7 & 30 & 17,3 & $(0,152 ; 0,717)$ & \\
\hline \multirow{2}{*}{$\begin{array}{l}\text { Frequentam lugares que os } \\
\text { pais desconhecem }\end{array}$} & Sim & 22 & 61,1 & 14 & 38,9 & 0,330 & \multirow{2}{*}{$0,004^{*}$} \\
\hline & Não & 143 & 82,7 & 30 & 17,3 & $(0,152 ; 0,717)$ & \\
\hline \multirow{2}{*}{ Pais ausentes } & Sim & 27 & 65,9 & 14 & 34,1 & 0,419 & \multirow{2}{*}{$0,022^{*}$} \\
\hline & Não & 138 & 82,1 & 30 & 17,9 & $(0,197 ; 0,894)$ & \\
\hline \multicolumn{8}{|c|}{ Problemas relacionados à escola } \\
\hline \multirow{2}{*}{ Faltar à escola } & Sim & 14 & 53,8 & 12 & 46,2 & 0,247 & \multirow{2}{*}{$0,001^{*}$} \\
\hline & Não & 151 & 82,5 & 32 & 17,5 & $(0,105 ; 0,584)$ & \\
\hline \multirow{2}{*}{ Irritabilidade durante a aula } & Sim & 44 & 65,7 & 23 & 34,3 & 0,332 & \multirow{2}{*}{$0,001^{*}$} \\
\hline & Não & 121 & 85,2 & 21 & 14,8 & $(0,167 ; 0,659)$ & \\
\hline \multicolumn{8}{|l|}{ Relacionamento com amigos } \\
\hline \multirow{2}{*}{$\begin{array}{l}\text { Uso regular de álcool ou } \\
\text { drogas por amigos }\end{array}$} & Sim & 34 & 58,6 & 24 & 41,4 & 0,216 & \multirow{2}{*}{$<0,001^{*}$} \\
\hline & Não & 131 & 86,8 & 20 & 13,2 & $(0,107 ; 0,437)$ & \\
\hline \multirow{2}{*}{$\begin{array}{l}\text { Comercialização de álcool ou } \\
\text { drogas por parte dos amigos }\end{array}$} & Sim & 23 & 57,5 & 17 & 42,5 & 0,257 & \multirow{2}{*}{$<0,001^{*}$} \\
\hline & Não & 142 & 84 & 27 & 16 & $(0,122 ; 0,545)$ & \\
\hline \multirow{2}{*}{$\begin{array}{l}\text { Desaprovação de amizades } \\
\text { por parte dos pais }\end{array}$} & Sim & 16 & 50 & 16 & 50 & 0,188 & \multirow{2}{*}{$<0,001^{*}$} \\
\hline & Não & 149 & 84,2 & 28 & 15,8 & $(0,084 ; 0,419)$ & \\
\hline \multirow{2}{*}{$\begin{array}{l}\text { Roubo ou dano à patrimônio } \\
\text { por parte dos amigos }\end{array}$} & Sim & 10 & 55,6 & 8 & 44,4 & 0,290 & \multirow{2}{*}{$0,017^{\dagger}$} \\
\hline & Não & 155 & 81,2 & 36 & 18,8 & $(0,107 ; 0,788)$ & \\
\hline \multirow{2}{*}{$\begin{array}{l}\text { Envolvimento com jovens } \\
\text { mais velhos }\end{array}$} & Sim & 22 & 56,4 & 17 & 43,6 & 0,244 & \multirow{2}{*}{$<0,001^{*}$} \\
\hline & Não & 143 & 84,1 & 27 & 15,9 & $(0,115 ; 0,520)$ & \\
\hline Lazer e recreação & & & & & & & \\
\hline Sair à noite sem & Sim & 8 & 57,1 & 6 & 42,9 & 0,323 & $04 \mathrm{Q}$ \\
\hline consentimento dos pais & Não & 157 & 80,5 & 38 & 19,5 & $(0,106 ; 0,985)$ & 0,049 ? \\
\hline Frequenta festas sem os & Sim & 37 & 63,8 & 21 & 36,2 & 0,317 & $0 \cap 001$ * \\
\hline pais & Não & 128 & 84,8 & 23 & 15,2 & $(0,158 ; 0,635)$ & 1, \\
\hline Uso de álcool ou drogas & Sim & 4 & 26,7 & 11 & 73,3 & 0,075 & $<0001+$ \\
\hline para diversão & Não & 161 & 83 & 33 & 17 & $(0,022 ; 0,248)$ & $<0,001$ \\
\hline
\end{tabular}

Fonte: Dados da pesquisa. *Teste de Qui-quadrado; †Teste Exato de Fisher

Tabela 4 - Classificação do estado nutricional antropométrico, peso, altura e IMC relacionado ao uso e não uso de álcool ( $n=209)$. Alfenas, MG, Brasil, 2013

\begin{tabular}{|c|c|c|c|c|c|c|c|}
\hline \multirow{3}{*}{ Classificação } & \multicolumn{4}{|c|}{ Uso de álcool } & \multicolumn{3}{|c|}{ Peso } \\
\hline & \multicolumn{2}{|c|}{ Não } & \multicolumn{2}{|c|}{ Sim } & \multirow{2}{*}{$\operatorname{Max}^{*}$} & \multirow{2}{*}{$\operatorname{Min}^{\dagger}$} & \multirow{2}{*}{$\mathrm{DP}^{\ddagger}$} \\
\hline & $\mathrm{n}$ & $\%$ & $\mathbf{n}$ & $\%$ & & & \\
\hline Desnutrido & 10 & 100 & 0 & 0 & 39,1 & 24,6 & 4,9 \\
\hline Eutrófico & 118 & 79,2 & 31 & 20,8 & 68 & 29,5 & 7,6 \\
\hline Sobrepeso & 26 & 74,3 & 9 & 25,7 & 69,7 & 39,2 & 7,4 \\
\hline Obesidade & 11 & 73,3 & 4 & 26,7 & 94,9 & 49 & 13,7 \\
\hline \multirow{2}{*}{ Classificação } & \multicolumn{4}{|c|}{ Altura } & \multicolumn{3}{|c|}{ IMC } \\
\hline & Max* & & & DP $\ddagger$ & $\operatorname{Max}^{*}$ & $\operatorname{Min}^{\dagger}$ & DP $\neq$ \\
\hline Desnutrido & 1,6 & & & 0,1 & 15 & 12 & 1,1 \\
\hline Eutrófico & 1,3 & & & 0,8 & 22 & 15 & 1,7 \\
\hline Sobrepeso & 1,7 & & & 0,1 & 27 & 20 & 1,5 \\
\hline Obesidade & 1,8 & & & 0,1 & 36 & 24 & 3 \\
\hline
\end{tabular}

Fonte: Dados da pesquisa. *Max - Máxima; †Min - Mínima; †DP - Desvio Padrão 


\section{Discussão}

O consumo de álcool entre adolescente é um sério problema social, político e de saúde(1). Embora exista, no Brasil, a lei em que proíbe a comercialização de bebidas alcoólicas para menores de 18 anos $^{(13)}$, os adolescentes estão se envolvendo com esta substância de forma cada vez mais precoce.

Neste estudo, 21\% dos estudantes entrevistados $(n=44)$ consumiam o álcool. Esse percentual foi semelhante ao obtido pela Pesquisa Nacional de Saúde do Escolar ${ }^{(14)}$, o qual verificou que $26,1 \%$ dos jovens brasileiros consumiram o álcool no último mês.

Esses dados revelam que o consumo de álcool por adolescentes brasileiros é uma realidade que merece atenção por se tratar de uma faixa etária em que os indivíduos se encontram em processo de intensas transformações e dúvidas, as quais acarretam mudanças biopsicossociais e cognitivas, sendo que tais transformações associado ao uso precoce do álcool favorecem a ocorrência de prejuízos nesses campos ${ }^{(3)}$. Além de ocasionar diversas alterações no adolescente, o uso do álcool é considerado porta de entrada para o consumo de drogas ilícitas ${ }^{(15)}$.

Pode-se observar que o consumo do álcool apresentou-se maior entre os alunos com idade superior a 13 anos e estes possuem mais chances de utilizarem o álcool, tais achados corroboram os resultados de outros estudos $^{(1,14-15)}$, em que as bebidas alcóolicas são mais consumidas pelos alunos mais velhos. Isto se deve ao ganho de autonomia e a necessidade dos jovens de se autoafirmarem, por conseguinte, isso favorece a participação em eventos sociais com bebidas alcoólicas de fácil acesso e, muitas vezes, na ausência de pais ou de responsáveis.

Por outro lado, no que tange ao sexo, neste estudo, meninas e meninos utilizaram essa substância na mesma proporção. Embora existam controvérsias a esse respeito(16), estudos têm mostrado um consumo homogêneo entre adolescentes do sexo feminino e masculino(13-14). Nota-se que a busca de igualdade entre gênero vem acarretando em mudanças de paradigmas em diversos aspectos, inclusive no que se refere ao consumo de álcool, que anteriormente prevalecia entre homens.

Em relação ao conhecimento dos alunos em torno dos prejuízos e danos causados pelo uso do álcool, a maioria dos adolescentes $(98,1 \%)$ relataram conhecer as consequências do uso dessa substância, o que também foi observado em outro estudo(4), em que os adolescentes reconhecem os riscos que o álcool acarreta à saúde, porém o utilizam por proporcionar bem-estar, para fugirem de problemas na vida pessoal e para facilitar a inserção no meio social.

Outro fator preocupante evidenciado no presente estudo refere-se a um maior risco dos indivíduos que consomem álcool se envolverem em acidentes automobilísticos, pois, como se sabe, o álcool altera de forma prejudicial a capacidade cognitiva do indivíduo, predispondo à ocorrência de acidentes, prevalecendo maior risco no sexo masculino(17).

No que se refere ao comportamento dos estudantes investigados neste estudo, aqueles que não utilizaram álcool apresentaram menor risco de desenvolver comportamentos inadequados. Esse resultado é condizente com os outros estudos em que os autores evidenciam que brigas entre os jovens, faltas à escola, atividade sexual desprotegida e situações conflituosas com familiares e amigos constituem consequências do consumo de bebidas alcóolicas ${ }^{(6-7)}$. Com isso, infere-se que o álcool influencia negativamente no comportamento desses adolescentes, encorajando-os a vivenciar situações de risco que acarretam consequências em curto e em longo prazo $^{(2)}$.

Outro achado importante deste estudo foi em relação a presença de pais ou de responsáveis em casa a maior parte do tempo e o fato de os mesmos conhecerem os ambientes que os filhos frequentam, o que conferiu um fator de proteção, diminuindo os riscos de os adolescentes se envolverem com bebidas alcoólicas, o que confirma o fato que o diálogo com os pais é um importante fator de proteção para se evitar o consumo de álcool(18).

Neste estudo, $48,8 \%$ dos alunos disseram que membros de sua família consomem bebidas alcoólicas, o que confere risco para esse adolescente iniciar o uso dessa substância, agravando o problema. Adicionalmente, em um estudo realizado com familiares holandeses, foi evidenciado que, quando ambos os pais consomem bebidas alcoólicas, os filhos terão maior chance de apresentarem uso excessivo dessa substância, quando os pais não bebem e estabelecem comunicação a respeito do consumo do álcool, as chances de consumo, por parte dos adolescentes, reduzem, conferindo um fator preventivo(5).

Ainda, este estudo demonstrou que os adolescentes que não ingeriram bebidas alcoólicas apresentaram três vezes menos chances de obterem problemas familiares, corroborando com o estudo(11) que apontou o consumo de drogas como fator desencadeador de problema no relacionamento familiar entre adolescentes. Portanto, o consumo de bebidas alcoólicas na adolescência pode estar associado ao fato de possuirem familiares que consomem tal substância e por vivenciarem situações coflituosas em meio familiar, como por exemplo, 
não possuir relacionamento amigável com os pais, sentimento de abandono e desobediência por parte dos adolescentes ${ }^{(11)}$.

Neste estudo, os adolescentes que não consumiram álcool possuem chances diminuídas de obterem problemas relacionados à amizades, assim como outro estudo(1) mostra que possuir amigos que utilizam bebida alcoólica, apresentam problemas com a lei, roubaram ou danificaram algo, vendem drogas, levam drogas a festas e ficam entediados quando não bebem em festas apresentou risco aumentado de usarem álcool e/ou outras drogas. Isso demonstra a influência dos amigos no comportamento do adolescente, em que muitas vezes os comportamentos são imitados, o que favorece tais atitudes inadequadas encorajadas pelo grupo, na busca pela popularidade.

Constatou-se neste estudo, por meio de correlações entre os dados, que não houve associação estatisticamente significativa entre o consumo de álcool e o estado nutricional antropométrico dos adolescentes. No entanto, não se deve ignorar uma possível relação entre essas variáveis, uma vez que outros pesquisadores $^{(8)}$ apontam a interferência do álcool no estado nutricional de seus consumidores, prevalecendo a influência de bebidas alcoólicas na obesidade abdominal, principalmente, no sexo masculino.

Apesar de as limitações do estudo estarem relacionadas ao modelo de investigação transversal, restringido a uma amostra de conveniência, os achados deste estudo contribuem para pontuar as áreas que requerem atenção dos profissionais de saúde, da família, da escola e da comunidade para o estabelecimento de estratégias de prevenção do consumo de álcool por adolescentes ou de redução de danos decorrentes do mesmo. Ainda, vale ressaltar a importância de investigações multicêntricas que envolvam vários estudantes, inclusive de outras séries do Ensino Fundamental e Médio de modo a ampliar o alcance dos resultados.

As pesquisas que apontam o perfil de consumo de álcool entre adolescentes, como ora apresentado, podem não retratar a verdadeira realidade brasileira, por possível dificuldade de adesão dos estudantes no preenchimento manual de instrumentos de pesquisa, que não despertam o interesse dos mesmos durante o processo de triagem; com isso, nota-se a necessidade de elaboração de ferramentas interativas e inovadoras a fim de despertar maior comprometimento e motivação nos jovens durante a coleta dos dados para a obtenção de resultados mais fidedignos.

\section{Conclusão}

O estudo permitiu concluir que o consumo de álcool pelos adolescentes de escola pública foi considerável, com o relato de uso aumentado para a idade de 13 anos ou mais e de forma homogênea entre os sexos.

Os adolescentes investigados que utilizaram álcool apresentaram maior probabilidade de se envolverem em situações de risco, como acidentes automobilísticos e dificuldades para resistir ao uso de outras drogas.

O álcool pode causar diversos efeitos negativos e prejudiciais à vida do adolescente e, somado a isso, surge a influência de amigos e de familiares para potencializar esse consumo. Como foi observado neste estudo, o fato de ter pais ou responsáveis presentes em casa, que conhecem o cotidiano e lugares em que os filhos frequentam e ter amigos que possuem bom comportamento, conferiram menores chances de consumirem bebidas alcoólicas.

Por conseguinte, torna-se relevante que os profissionais de saúde, com destaque o enfermeiro, formulem estratégias para afastar os jovens dos fatores de risco que aumentam a probabilidade de envolvimento com o álcool, e potencializar os fatores protetores que contribuem para a redução das chances de uso.

\section{Referências}

1. Cardoso LRD, Malbergier A. A influência dos amigos no consumo de drogas entre adolescentes. Estud Psicol. 2014;31(1):65-73.

2. Pierobon M, Barak M, Hazrati S, Jacobsen KH. Alcohol consumption and violence among Argentine adolescents. J Pediatr. 2013; 89 (1):100-7.

3. Silva MHN, Ávilla AL, Alves LSR, Reis IR, Rafael JC. Atenção básica e o uso de álcool e drogas por adolescentes: prevenção e conduta. Rev Gestão Saúde. [internet]. 2013; [Acesso 4 fev 2015]; 4(2):317-36. Disponível em: http:// periodicos.unb.br/index.php/rgs/article/view/22948/16471 4. Silva SED, Padilha MI. Alcoholism in adolescents' life histories: an analysis in the light of social representations. Texto Contexto Enferm. 2013 jul-set; 22 (3):576-84.

5. Mares SHW, van der Vorst H, Engels RC, LichtwarckAschoff A. Parental alcohol use, alcohol-related problems, and alcohol-specific attitudes, alcohol-specific communication, and adolescent excessive alcohol use and alcohol-related problems: An indirect path model. Addict Behav [Internet]. 2011 mar; [cited 2015 Mar 14]; 36(3):20916. Available from: http://www.sciencedirect.com/science/ article/pii/S0306460310003011. 
6. Larrosa SL, Palomo JLRA. Factores de riesgo y de protección en el consumo de drogas en adolescentes y diferencias según edad y sexo. Psicothema. [Internet]. 2010; [Acceso 22 mar 2015]; 22(4):568-73. Disponible en: http://www.redalyc.org/articulo.oa?id=72715515007.

7. Sampaio FJL Filho, Sousa PRM, Vieira NFC, Nóbrega MFB, Gubert FA, Pinheiro PNC. Percepção de risco de adolescentes escolares na relação consumo de álcool e comportamento sexual. Rev Gaúcha Enferm. set 2010;31(3):508-14.

8. Guimarães NS, Nemer ASA, Fausto MA. Influência do consumo de álcool nas alterações antropométricas: uma revisão sistemática. Nutr Clín Diet Hosp. [Internet]. 2013; [Acesso 22 mar 2015]; 33(3): 68-73. Disponível em: http:// revista.nutricion.org/PDF/333sernizon.pdf.

9. Tarter RE. Evaluation and treatment of adolescent substance abuse: a decision tree method. Am J Drug Alcohol Abuse. 1990;16(1/2):1-46.

10. De Micheli D, Formigoni MLOS. Screening of drug use in a teenage brazilian sample using the Drug Use Screening Inventory (DUSI). Addict Behav. 2000;25:683-91.

11. Malbergier A, Cardoso LRD, Amaral RA. Uso de substâncias na adolescência e problemas familiares. Cad Saúde Pública. 2012;28(4):678-88.

12. Ministério da Saúde (BR), Coordenação Geral da Política de Alimentação e Nutrição. Sistema de Vigilância Alimentar e Nutricional - SISVAN: Incorporação das Curvas de Crescimento da Organização Mundial de Saúde de 2006 e 2007 no SISVAN. [Internet] 2007; [Acesso 21 mar 2015]. Disponível em: http://dab.saude.gov.br/portaldab/ ape_vigilancia_alimentar.php?conteudo=curvas_de_ crescimento.

13. Dallo L. Martins RA. Uso de álcool entre adolescentes escolares: um estudo-piloto. Paidéia. [Internet]. set-dez 2011; [Acesso 20 mar 2015]; 21(50); [aprox. 6 telas]. Disponível em: http:/www.scielo.br/paideia.

14. Instituto Brasileiro de Geografia e Estatística (IBGE). Pesquisa Nacional de Saúde do Escolar (PeNSE). [Internet]. Rio de Janeiro: 2013. [Acesso 22 mar 2015]; Disponível em: http://www.ibge.gov.br/home/estatistica/ populacao/pense/2012/pense_2012.pdf.

15. Simões C, Matos MG, Batista-Foguet, JM, SimonsMorton B. Substance Use Across Adolescence: Do Gender and Age Matter? Psicol Reflex Crit. 2014;27(1):179-88.

16. Rozin L, Zagonel IPS. Risk factors for alcohol dependence in adolescents. Acta Paul Enferm. 2012;25(2):314-8.
17. Voas RB, Torres P, Romano E, Lacey JH. AlcoholRelated Risk of Driver Fatalities:An Update Using 2007 Data. J Stud Alcohol Drugs. 2012 May; 73(3):341-50.

18. Atanázio EA, Santos J M, Dionísio LR, Silva J, Saldanha AAW. Vulnerability to alcohol use: study with adolescentes from the public and private school systems. SMAD, Rev. Eletrônica Saúde Mental Álcool Drog. [Internet]. 2013 jan; 9(1):11-7. Disponível em: http://pepsic. bvsalud.org/scielo.php?script=sci_arttext\&pid=S1806$69762013000100003 \&$ Ing=pt\&nrm=iso 